\title{
Representations of Central Extensions of Differentiably Simple Lie Superalgebras
}

\author{
Shun-Jen Cheng \\ Department of Mathematics, Harvard University, Cambridge, MA 02138, U.S.A.
}

Received September 29, 1992

\begin{abstract}
Let $S$ be a simple Lie algebra of characteristic 0 and $\Lambda(n)$ be the Grassmann superalgebra in $n$ indeterminates. We can form the Lie superalgebra $S \otimes \Lambda(n)$. The purpose of this paper is to classify all finite dimensional irreducible representations of all central extensions of $S \otimes \Lambda(n)$. We will also give a character formula for these representations.
\end{abstract}

\section{Introduction}

A finite dimensional differentiably simple Lie superalgebra is a Lie superalgebra that has no nontrivial differential ideals. In [K1] Kač suggested using methods developed in [B] to show that every such Lie superalgebra is the tensor product of a simple Lie superalgebra and a Grassmann superalgebra. It is not very hard to show that their finite dimensional irreducible representations are just irreducible representations of the simple Lie superalgebra with the unique maximal ideal acting trivially. A proof of this can be found in [C]. In this paper we will study central extensions of the Lie superalgebra $S \otimes \Lambda(n)$, where $S$ is a simple Lie algebra. We will explicitly construct central extensions and use this to obtain a character formula for finite dimensional irreducible representations of such extensions.

We call the tensor product of the Laurent polynomials (in several, say $m$, variables) and a Grassmann superalgebra the superalgebra of super Laurent polynomials. Now let $S$ be a finite dimensional simple Lie superalgebra. The general loop superalgebra associated to $S$ is obtained by taking the tensor product of $S$ and the super Laurent polynomials. Following the definition of the current (= affine Kač-Moody algebra) algebra, we call the central extensions of the general loop superalgebra the general current superalgebra. This paper deals with the special case when $S$ is a Lie algebra and $m=0$. It is our hope that it can shed some light on the representation theory of the general case.

This paper is organized as follows: In Sect. 1 we prove a $1-1$ correspondence between central extensions and "superskewsymmetric derivations." To do this we apply a technique used in the construction of the current algebra starting with the loop algebra. This method is due to Kač, and sketches of it can be found in some of 
the exercises in [K2]. In Sect. 2 we show that only certain superskewsymmetric derivations actually give rise to interesting finite dimensional representations. Sections 3 and 4 show that the usual integrability condition for the unique irreducible highest weight representation to be finite dimensional is not sufficient. Finally in Sect. 5 we determine under what condition the irreducible highest weight representations are actually finite dimensional. We will also construct these representations explicitly, using the idea of "minimal representations," which can be found in [K3].

\section{Central Extensions}

$k$ will stand for an algebraically closed field of characteristic 0 . Throughout this paper when $\operatorname{deg} a$ appears in an expression, we assume that $a$ is an element, homogeneous of degree $\operatorname{deg} a$, in a $\mathbb{Z}_{2}$-graded space. Let $V=V_{\overline{0}} \oplus V_{\overline{1}}$ be a $\mathbb{Z}_{2}$ graded vector space over $k$ and let $(\cdot \mid \cdot): V \times V \mapsto k$ be a bilinear form. $(\cdot \mid \cdot)$ is said to be supersymmetric if $(x \mid y)=(-1)^{(\operatorname{deg} x)(\operatorname{deg} y)}(y \mid x)$. It is said to be superskewsymmetric if $(x \mid y)=-(-1)^{(\operatorname{deg} x)(\operatorname{deg} y)}(y \mid x)$. Furthermore $(\cdot \mid \cdot)$ will be called consistent if $\left(V_{\varepsilon} \mid V_{\delta}\right)=0$ for $\varepsilon \neq \delta \in \mathbb{Z}_{2}$; and anti-consistent if $\left(V_{\varepsilon} \mid V_{\delta}\right)=0$ for $\varepsilon=\delta \in \mathbb{Z}_{2}$.

Suppose now that $(\cdot \mid \cdot)$ is nondegenerate. Let $T \in \operatorname{End}_{k}(V)$. The adjoint of $T$, denoted by $T^{*}$, is the (unique) element in $\operatorname{End}_{k}(V)$ satisfying

$$
(T(x) \mid y)=(-1)^{(\operatorname{deg} T)(\operatorname{deg} x)}\left(x \mid T^{*}(y)\right) \quad \forall x, y \in V .
$$

$T$ will be called supersymmetric if $T^{*}=T$, and superskewsymmetric if $T^{*}=-T$.

Let $L$ be a Lie superalgebra. A central extension $\hat{L}$ of $L$ (by the even central element $z$ ) is an exact sequence of Lie superalgebra homomorphisms

$$
0 \mapsto k z \stackrel{i}{\mapsto} \hat{L} \stackrel{\rho}{\mapsto} L \mapsto 0
$$

So $\hat{L} \cong L \oplus k z$ as vector spaces. We will identify $\hat{L}$ with $L+k z$. Let $x, y \in L \subseteq \hat{L}$. Let's denote the bracket of the Lie superalgebra $L$ by $[\cdot, \cdot]_{0}$, and the bracket of the Lie superalgebra $\hat{L}$ by $[\cdot, \cdot]$. Then the exact sequence above is equivalent to the existence of a bilinear form $\alpha$, called the 2-cocycle, such that

$$
[x, y]=[x, y]_{0}+\alpha(x, y) z, \quad \forall x, y \in L .
$$

It follows that $\alpha$ is superskewsymmetric and satisfies the 2-cocycle condition

$$
\alpha(x,[y, z])=\alpha([x, y], z)+(-1)^{(\operatorname{deg} x)(\operatorname{deg} y)} \alpha(y,[x, z]), \quad \forall x, y, z \in L .
$$

Two extensions $\hat{L}$ and $\hat{L}^{\prime}$ are equivalent if there exists an isomorphism of Lie superalgebras $\psi: \hat{L} \mapsto \hat{L}^{\prime}$ such that the following diagram commutes:

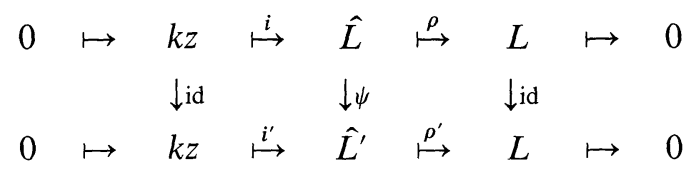

Let $L$ be a Lie superalgebra endowed with a nondegenerate supersymmetric invariant bilinear form $(\cdot \mid \cdot)$. Assume furthermore that $(\cdot \mid \cdot)$ is either consistent or anti-consistent. Let $D \in \operatorname{der}_{k} L$ be a homogeneous superskewsymmetric derivation, i.e. $D^{*}=-D$. We can construct a central extension of $L$ as follows: define 
a 2-cocycle $\alpha_{D}$, corresponding to $D$, by

$$
\alpha_{D}(x, y)=(D(x) \mid y) .
$$

$\alpha_{D}$ is superskewsymmetric, since $D$ is superskewsymmetric. The invariance of $(\cdot \mid \cdot)$ and the fact that $D$ is a derivation imply that $\alpha_{D}$ satisfies the 2-cocycle condition.

On the other hand if $\alpha$ is a 2-cocycle, then $\alpha(x, y)=(D(x) \mid y)$ for some $D \in \operatorname{End}_{k}(L)$ by the nondegeneracy of $(\cdot \mid \cdot)$. Since $\alpha$ is superskewsymmetric, $D$ is superskewsymmetric. Furthermore it is easy to check that the 2-cocycle condition implies that $D$ is a derivation.

Now by the invariance of $(\cdot \mid \cdot)$ ad $x, x \in L$, is a superskewsymmetric derivation. Let $\hat{L}$ denote the central extension corresponding to ad $x$. Let $L^{\prime}=L+k z$ denote the trivial central extension of $L$. The invariance of $(\cdot \mid \cdot)$ implies that the map $\psi: L^{\prime} \mapsto \hat{L}$ given by $\psi(y)=y+(x \mid y) z, y \in L$, and $\psi(z)=z$ is an isomorphism of Lie superalgebras.

Conversely, suppose that $\hat{L}$ is the central extension corresponding to some derivation $D$ such that $\hat{L}$ is equivalent to the trivial extension $L^{\prime}$. Thus we have a Lie superalgebra isomorphism $\psi: L^{\prime} \mapsto \hat{L}$ such that $\psi(z)=z$ and $\psi(y)=y+\beta(y), \forall y \in L$ and $\beta \in L^{*}$. Since $(\cdot \mid \cdot)$ is nondegenerate, there exists $x \in L$ such that $\beta(y)=(x \mid y), \forall y \in L$. It is easy to check, using invariance and the fact that $\psi$ preserves the bracket, that $D=\operatorname{ad} x$. We conclude that there exists a 1-1 correspondence between nontrivial central extensions of $L$ and outer derivations of $L$.

We will apply the correspondence obtained above to the Lie superalgebra $L=S \otimes \Lambda(n)$, where $S$ is a simple Lie algebra. We let $\Lambda(n)$ have the indeterminates $\xi_{1}, \xi_{2}, \ldots, \xi_{n}$ and $(\cdot 1 \cdot)_{0}$ be a symmetric nondegenerate invariant bilinear form of $S$. We define a supersymmetric bilinear form on $L$ by

$$
\left(s_{1} \otimes \lambda_{1} \mid s_{2} \otimes \lambda_{2}\right)=\left(s_{1} \mid s_{2}\right)_{0} \int \lambda_{1} \wedge \lambda_{2},
$$

where $s_{1}, s_{2} \in S ; \lambda_{1}, \lambda_{2} \in \Lambda(n)$ and $\int \lambda$ is the $\left(\xi_{1} \wedge \xi_{2} \wedge \ldots \wedge \xi_{n}\right)^{\text {th }}$ coefficient of $\lambda \in \Lambda(n)$, called the base number of $\lambda$. Clearly $(\cdot \mid \cdot)$ is nondegenerate. Invariance follows immediately from the invariance of $(\cdot \mid \cdot)_{0}$. Also if $n$ is even, then $(\cdot \mid \cdot)$ is consistent; and if $n$ is odd, then $(\cdot \mid \cdot)$ is anti-consistent.

The derivation algebra of $A \otimes B$, where $A$ is an algebra with $A^{2}=A$ and $B$ is a unitary commutative associative algebra, is shown in [B] to be isomorphic to $\operatorname{der}_{k} A \otimes B+C(A) \otimes \operatorname{der}_{k} B$, where $C(A)$ is the centroid of $A$. Similar arguments can be used to show that this is true for superalgebras. Therefore we have $\operatorname{der}_{k}(S \otimes \Lambda(n)) \cong S \otimes \Lambda(n)+W(n)$, where $W(n)$ stands for the derivation superalgebra of $\Lambda(n)$. So nontrivial central extensions are obtained by using homogeneous superskewsymmetric derivations of $\Lambda(n)$.

Remark. Since the central element $z$ is even, only even superskewsymmetric elements of $W(n)$ give rise to central extensions in the case when $n$ is even. Similarly, when $n$ is odd, only odd superskewsymmetric elements of $W(n)$ need to be considered.

Remark. It is not hard to see that every element $D \in W(n)$ has the form $D=\sum_{i=1}^{n} P_{i} \wedge \frac{\partial}{\partial \xi_{i}}$, where $P_{i} \in \Lambda(n)$ and $\frac{\partial}{\partial \xi_{i}}$ is the derivation of $\Lambda(n)$ such that $\frac{\partial}{\partial \xi_{i}}\left(\xi_{j}\right)=\delta_{i j}($ see $[\mathrm{K} 1])$. 
Lemma 1.1. Let $a, b \in \Lambda(n)$ and $D \in W(n)_{-1}$. Then

$$
\int D(a) \wedge b=-(-1)^{\operatorname{deg} a} \int a \wedge D(b) .
$$

Proof. By linearity of $\int$ we can assume that $a$ and $b$ are both homogeneous in the $\mathbb{Z}$-grading of $\Lambda(n)$. Since $D$ is an odd derivation, we have

$$
\int D(a \wedge b)=\int D(a) \wedge b+(-1)^{\operatorname{deg} a} \int a \wedge D(b) .
$$

The left-hand side is always 0 , since $D \in W(n)_{-1}$.

Proposition 1.1. $D=\sum_{i=1}^{n} P_{i} \wedge \frac{\partial}{\partial \xi_{i}}, P_{i} \in \Lambda(n)$, is superskewsymmetric if and only if $\sum_{i=1}^{n} \frac{\partial}{\partial \xi_{i}}\left(P_{i}\right)=0$

Proof. Let $a$ and $b$ be homogeneous elements of $\Lambda(n)$. Then

$$
\begin{aligned}
\int D(a) \wedge b & =\int \sum_{i=1}^{n} P_{i} \wedge \frac{\partial}{\partial \xi_{i}}(a) \wedge b \\
= & \sum_{i=1}^{n}(-1)^{\left(\operatorname{deg} P_{\imath}\right)(\operatorname{deg} a)+\left(\operatorname{deg} P_{i}\right)} \int \frac{\partial}{\partial \xi_{i}}(a) \wedge P_{i} \wedge b \\
= & -\sum_{i=1}^{n}(-1)^{\left(\operatorname{deg} P_{i}\right)(\operatorname{deg} a)+\left(\operatorname{deg} P_{i}\right)+(\operatorname{deg} a)} \int a \wedge \frac{\partial}{\partial \xi_{i}}\left(P_{i} \wedge b\right) \quad(\text { by Lemma 1.1) } \\
= & -\sum_{i=1}^{n}(-1)^{\left(\operatorname{deg} P_{i}\right)(\operatorname{deg} a)+\left(\operatorname{deg} P_{i}\right)+(\operatorname{deg} a)} \int a \wedge \frac{\partial}{\partial \xi_{i}}\left(P_{i}\right) \wedge b \\
& -\sum_{i=1}^{n}(-1)^{\left(\operatorname{deg} P_{\imath}\right)(\operatorname{deg} a)+(\operatorname{deg} a)} \int a \wedge P_{i} \wedge \frac{\partial}{\partial \xi_{i}}(b) \\
= & -\sum_{i=1}^{n}(-1)^{\operatorname{deg} P_{i}} \int \frac{\partial}{\partial \xi_{i}}\left(P_{i}\right) \wedge(a \wedge b) \\
= & -(-1)^{(\operatorname{deg} D)(\operatorname{deg} a)} \int a \wedge D(b) .
\end{aligned}
$$

Hence $D$ is superskewsymmetric if and only if

$$
\sum_{i=1}^{n} \int \frac{\partial}{\partial \xi_{i}}\left(P_{i}\right) \wedge(a \wedge b)=0, \quad \forall a, b \in \Lambda(n)
$$

This is true if and only if $\sum_{i=1}^{n} \frac{\partial}{\partial \xi_{i}}\left(P_{i}\right)=0$.

Summarizing, we have established a 1-1 correspondence between nontrivial central extensions of $S \otimes \Lambda(n)$ and elements in $W(n)$ with zero divergence. We will call these derivations central derivations.

\section{Representations of $\hat{L}_{D}$ with $D \notin W(n)_{n-2}$}

Write $\Lambda(n)=\bigoplus_{j \in \mathbb{Z}} \Lambda(n)_{j}$ and $W(n)=\bigoplus_{j \in \mathbb{Z}} W(n)_{j}$, where $\Lambda(n)_{j}$ and $W(n)_{j}$ denote the homogeneous (in their natural $\mathbb{Z}$-grading) subspaces of degree $j$ in $\Lambda(n)$ and $W(n)$, respectively. Furthermore let $N$ be the unique maximal ideal of $\Lambda(n)$. 
Lemma 2.1. Let $D \in W(n)$. Suppose there exists $a \lambda \in \Lambda(n)_{j}, j \geqq 2$, such that $D(\lambda) \neq 0$. Then there exists a $\lambda^{\prime} \in \Lambda(n)_{j-1}$ such that $D\left(\lambda^{\prime}\right) \neq 0$.

Proof. It suffices to prove the lemma for $D \in W(n)_{k}$, for some $k \in \mathbb{Z}$. Let $D=\sum_{i=1}^{n} \lambda_{i} \wedge \frac{\partial}{\partial \xi_{i}}, \lambda_{i} \in \Lambda(n)_{k+1}$. We may assume, after relabeling $\xi_{1}, \xi_{2}, \ldots, \xi_{n}$ if necessary, that $D\left(\xi_{1} \wedge \xi_{2} \wedge \ldots \wedge \xi_{j}\right) \neq 0$. Now

$$
\begin{aligned}
D\left(\xi_{1} \wedge \xi_{2} \wedge \ldots \wedge \xi_{j}\right) & =\sum_{i=1}^{n} \lambda_{i} \wedge \frac{\partial}{\partial \xi_{i}}\left(\xi_{1} \wedge \xi_{2} \wedge \ldots \wedge \xi_{j}\right) \\
& =\sum_{i=1}^{j} \lambda_{i} \wedge \frac{\partial}{\partial \xi_{i}}\left(\xi_{1} \wedge \xi_{2} \wedge \ldots \wedge \xi_{j}\right) \\
& =\sum_{i=1}^{j}(-1)^{i-1} \lambda_{i} \wedge \xi_{1} \wedge \ldots \xi_{i-1} \wedge \xi_{i+1} \ldots \wedge \xi_{j} .
\end{aligned}
$$

By assumption we have a nonzero sum of $j$ vectors. This implies that there is a nonzero sum of $j-1$ vectors, since char $k=0$. (If not, then the sum over all sums of $j-1$ vectors would be zero.) Now we may assume, after relabeling $\xi_{1}, \xi_{2}, \ldots, \xi_{j}$ if necessary, that

$$
\sum_{i=1}^{j-1}(-1)^{i-1} \lambda_{i} \wedge \xi_{1} \wedge \ldots \xi_{i-1} \wedge \xi_{i+1} \ldots \wedge \xi_{j} \neq 0 .
$$

Hence

$$
\left(\sum_{i=1}^{j-1}(-1)^{i-1} \lambda_{i} \wedge \xi_{1} \wedge \ldots \xi_{i-1} \wedge \xi_{i+1} \ldots \wedge \xi_{j-1}\right) \wedge \xi_{j} \neq 0,
$$

which implies that

$$
\sum_{i=1}^{j-1}(-1)^{i-1} \lambda_{i} \wedge \xi_{1} \wedge \ldots \xi_{i-1} \wedge \xi_{i+1} \ldots \wedge \xi_{j-1} \neq 0 .
$$

But this expression is $D\left(\xi_{1} \wedge \xi_{2} \wedge \ldots \wedge \xi_{j-1}\right)$.

This simple lemma is crucial, since we are only interested in finite dimensional representations of central extensions of $S \otimes \Lambda(n)$. Proposition 1.1 tells us that in order to construct central extensions, we only need to consider central derivations of $\Lambda(n)$. There are many such derivations for big $n$ 's. However, Lemma 2.1 will "eliminate" most of them, as they do not give rise to interesting representations, as we shall see below.

Let $L=S \otimes \Lambda(n)$ and let $(\cdot \mid \cdot)_{0}$ be a nondegenerate invariant symmetric bilinear form of $S$. Let $\hat{L}_{D}$ denote the central extension of $L$ associated to the central derivation $D \in W(n)$. As before we will denote the bracket of $S$ by $[\cdot \mid \cdot]_{0}$ and the bracket of $\hat{L}_{D}$ by $[\cdot \mid \cdot]$. For $s_{1}, s_{2} \in S$ and $\lambda_{1}, \lambda_{2} \in \Lambda(n)$ one has

$$
\left[s_{1} \otimes \lambda_{1}, s_{2} \otimes \lambda_{2}\right]=\left[s_{1}, s_{2}\right]_{0} \otimes \lambda_{1} \wedge \lambda_{2}+\left(s_{1} \mid s_{2}\right)_{0} \int D\left(\lambda_{1}\right) \wedge \lambda_{2} \text {. }
$$

Choose $\mathfrak{h} \subseteq S$ a Cartan subalgebra. Let $\mathfrak{h} \subseteq \mathfrak{b}$ be a Borel subalgebra of $S$. We let $\hat{\mathfrak{b}}=S \otimes N+\mathfrak{b}+k z$.

Lemma 2.2. Suppose that $0 \neq D \in W(n)_{k}, k<n-2$, is a central derivation. Then $z \in\left[\hat{\mathfrak{b}}_{\overline{1}}, \hat{\mathfrak{b}}_{\overline{1}}\right] \cap\left[\hat{\mathfrak{b}}_{\overline{0}}, \hat{\mathfrak{b}}_{\overline{0}}\right]$. 
Proof. Since $D \neq 0$, there exists $\xi_{i}$ such that $D\left(\xi_{i}\right) \neq 0$ for some $i$. Let $\lambda \in \Lambda(n)_{n-k-1}$ such that

$$
D\left(\xi_{i}\right) \wedge \lambda=\xi_{1} \wedge \xi_{2} \wedge \ldots \wedge \xi_{n} .
$$

By superskewsymmetry of $D, D(\lambda) \neq 0$. Now $k<n-2$ implies that $n-k-1 \geqq 2$. Hence by Lemma 2.1 there exists $\lambda^{\prime} \in \Lambda(n)_{n-k-2}$ such that $D\left(\lambda^{\prime}\right) \neq 0$. Hence there exists $\mu \in \Lambda(n)_{2}$ such that

$$
D\left(\lambda^{\prime}\right) \wedge \mu=\xi_{1} \wedge \xi_{2} \wedge \ldots \wedge \xi_{n} .
$$

Now choose $h_{1}, h_{2} \in \mathfrak{h}$ such that $\left(h_{1} \mid h_{2}\right)_{0} \neq 0$. Then

$$
\begin{aligned}
& z=\frac{1}{\left(h_{1} \mid h_{2}\right)_{0}}\left[h_{1} \otimes \xi_{i}, h_{2} \otimes \lambda\right] \in\left[\hat{\mathfrak{b}}_{\overline{1}}, \hat{\mathfrak{b}}_{\overline{1}}\right] \quad \text { and } \\
& z=\frac{1}{\left(h_{1} \mid h_{2}\right)_{0}}\left[h_{1} \otimes \lambda^{\prime}, h_{2} \otimes \mu\right] \in\left[\hat{\mathfrak{b}}_{\overline{0}}, \hat{\mathfrak{b}}_{\overline{0}}\right] . \quad \square
\end{aligned}
$$

Proposition 2.1. Let $(\pi, V)$ be a finite dimensional irreducible representation of $\hat{L}_{D}$. Suppose that $D \notin W(n)_{n-2}$. Then $\pi(z)=0$, and hence $V$ is an irreducible S-module.

Proof. Suppose that $D=D_{k}+D_{k-2}+\cdots$ with $0 \neq D_{i}$ for some $i<n-2$. Let $\hat{\mathrm{b}}$ be as before. Clearly $\hat{\mathfrak{b}}$ is solvable. Now by Lemma 2.1 we can use $D_{i}$ to conclude that $z \in\left[\hat{\mathfrak{b}}_{\overline{0}}, \hat{\mathfrak{b}}_{\overline{0}}\right]$. Therefore

$$
\left[\hat{\mathfrak{b}}_{\overline{1}}, \hat{\mathfrak{b}}_{\overline{1}}\right] \subseteq\left[\mathrm{b}, \hat{\mathfrak{b}}_{\overline{0}}\right]+k z \subseteq\left[\hat{\mathfrak{b}}_{\overline{0}}, \hat{\mathfrak{b}}_{\overline{0}}\right] .
$$

Thus by a theorem of Kač in [K1] all finite dimensional irreducible $\hat{b}$-modules are 1 -dimensional. Therefore $z$ acts trivially on all such $\hat{b}$-modules. Since $z$ is central, $z$ acts trivially on finite dimensional irreducible $\hat{L}_{D}$-modules. The fact that $V$ is an irreducible $S$-module follows from the fact that the finite dimensional irreducible representations of $S \otimes \Lambda(n)$ are just irreducible representations of $S$ (see [C]).

Proposition 2.1 classifies all finite dimensional irreducible representations of $\hat{L}_{D}$ in the case when $D \notin W(n)_{n-2}$. The remaining sections of this paper deal with the more interesting case when $D \in W(n)_{n-2}$.

\section{Irreducible Highest Weight Representations of $\hat{L}_{D}$}

Recall some of our notations from previous sections: $S$ is a simple Lie algebra with a nondegenerate symmetric invariant bilinear form $(\cdot \mid \cdot)_{0}$ and $L=S \otimes \Lambda(n)$. If $D$ is a central derivation, then $\hat{L}_{D}$ denotes the central extension (by the central element $z$ ) of $L$ associated to $D$. In this section we will begin our study of irreducible representations of the Lie superalgebra $\hat{L}_{D}$, where $D$ is homogeneous of degree $n-2$ in the $\mathbb{Z}$-graded Lie superalgebra $W(n)$. We only need to consider the case when the central element $z$ acts as a nonzero scalar $c \in k$.

From now on $D$ stands for a (fixed) central derivation, homogeneous of degree $n-2$ in the $\mathbb{Z}$-grading of $W(n)$. We let $\mathfrak{h}$ be a Cartan subalgebra of $S$ and $\Delta$ the set of roots in $\mathfrak{h}^{*}$. Denote by $\Delta_{+}$a set of positive roots and $\Pi=\left\{\alpha_{1}, \alpha_{2}, \ldots, \alpha_{r}\right\}$ the set of simple roots. Furthermore we will write $Q_{+}$for the positive root lattice and $\mathfrak{b}$ for the Borel subalgebra $\mathfrak{h}+\mathfrak{n}_{+}$, where $\mathfrak{n}_{+}$is the nilpotent subalgebra corresponding to $\Delta_{+}$. For each simple root $\alpha_{i}$, let $\left\{H_{i}, E_{i}, F_{i}\right\}$ be the corresponding copy of $s l_{2}(k)$ in $S$. 
Consider the solvable subalgebra $\hat{K}_{D}=k z+\mathfrak{b}+\sum_{j>1} S \otimes \Lambda(n)_{j} . \hat{K}_{D}$ is a subalgebra of $\hat{L}_{D}$ and satisfies Kač's solvability condition. Hence $\sum_{j>1} S \otimes \Lambda(n)_{j}$ acts trivially on any finite dimensional irreducible representation of $\hat{K}_{D}$. Now $\sum_{j>1} S \otimes \Lambda(n)_{j}$ is an ideal of $\hat{L}_{D}$, hence it must act trivially on any finite dimensional irreducible $\hat{L}_{D}$-module. So in order to describe finite dimensional irreducible representations of $\hat{L}_{D}$, it will suffice to consider irreducible representations of the Lie superalgebra $S+S \otimes \Lambda(n)_{1}+k z$ with

$$
\begin{aligned}
{\left[s_{1} \otimes 1, s_{2} \otimes \lambda\right] } & =\left[s_{1}, s_{2}\right] \otimes \lambda \text { and } \\
{\left[s_{1} \otimes \lambda_{1}, s_{2} \otimes \lambda_{2}\right] } & =\alpha_{D}\left(s_{1} \otimes \lambda_{1}, s_{2} \otimes \lambda_{2}\right) z=\left(\left(s_{1} \mid s_{2}\right)_{0} \int D\left(\lambda_{1}\right) \wedge \lambda_{2}\right) z,
\end{aligned}
$$

where $\lambda \in k+\Lambda(n)_{1} ; \lambda_{1}, \lambda_{2} \in \Lambda(n)_{1}$ and $s_{1}, s_{2} \in S$. We will denote this Lie superalgebra also by $L_{D}$. Since we are only interested in its finite dimensional irreducible representations, this should not cause serious problems.

Now let $I_{D} \subseteq S \otimes \Lambda(n)_{1}$ be a maximal isotropic subspace (with respect to the symmetric bilinear form $\alpha_{D}$ ) containing $n_{+} \otimes \Lambda(n)_{1}$. Consider the subalgebra $\hat{\mathfrak{b}}_{D}=\mathfrak{b}+I_{D}+k z \subseteq \hat{L}_{D}$. Since $\left[I_{D}, I_{D}\right]=0$, every finite dimensional irreducible representation of $\hat{\mathfrak{b}}_{D}$ is 1 -dimensional. Let $v_{A}$ be such a representation. Clearly $v_{A}$ is just a representation of $\mathfrak{h}+k z$ with $I_{D}$ acting trivially on $v_{\Lambda}$. Suppose that

$$
h v_{\Lambda}=\Lambda(h) v_{\Lambda}, h \in \mathfrak{h}, \Lambda \in \mathfrak{h}^{*} \quad \text { and } \quad z v_{\Lambda}=c v_{\Lambda}, 0 \neq c \in k .
$$

The induced module $\hat{V}_{D}(\Lambda, c)=\operatorname{Ind}_{\hat{\mathfrak{L}}_{D}}^{\hat{L}_{D}} v_{\Lambda}$ is a direct sum of weight spaces of $\mathfrak{h}$ with highest weight $\Lambda$, i.e. we have

$$
\operatorname{Ind}_{\hat{\mathfrak{b}}_{D}}^{\hat{L}_{D}} v_{\Lambda}=\bigoplus_{\mu \in \Lambda-Q_{+}} V_{\mu},
$$

where $V_{\mu}=\left\{x \in \operatorname{Ind}_{\hat{b}_{D}}^{\hat{L}_{D}} v_{\Lambda} \mid h x=\mu(h) x, \forall h \in \mathfrak{h}\right\}$.

By standard arguments of representation theory if $M$ is an $\hat{L}_{D}$-submodule, then

$$
M=\bigoplus_{\mu \in \Lambda-Q_{+}} V_{\mu} \cap M
$$

Also any $\hat{L}_{D}$-submodule containing a nonzero element of $V_{A}$ necessarily contains the vector $v_{\Lambda}$, since $I_{D}$ is maximal isotropic. Thus $\operatorname{Ind}_{\hat{b}_{D}} v_{\Lambda}$ has a unique maximal submodule $\hat{M}_{D}(\Lambda, c)$, and so it has a unique irreducible quotient. We will denote this quotient by $\hat{L}_{D}(\Lambda, c)$. It is not hard to see that if $\hat{L}_{D}(\Lambda, c)$ is finite dimensional, then $\Lambda\left(H_{i}\right) \in \mathbb{Z}_{+}$for $i=1, \ldots, r$. (This integrability condition however is by no means sufficient, as we will see in the next section.) Finally, by the usual arguments of highest weight representations two $\hat{L}_{D}$-modules $\hat{L}_{D}\left(\Lambda_{1}, c\right)$ and $\hat{L}_{D}\left(\Lambda_{2}, c\right)$ are isomorphic if and only if $\Lambda_{1}=\Lambda_{2}$.

\section{The Infinite Dimensionality of $\hat{L}_{D}(\Lambda, c)$ for $\Lambda \gtreqless r \rho$}

We will continue to use the notations defined in the previous sections. Let $D$ be a central derivation of degree $n-2$ in $W(n)$. Let $\bar{D}: \Lambda(n)_{1} \mapsto \Lambda(n)_{n-1}$ be the restriction of $D$ to $\Lambda(n)_{1}$, the subspace of $\Lambda(n)$ of degree 1 . We have shown in the previous section that the representation theory of $\hat{L}_{D}$ reduces to the representation theory of the quotient superalgebra $S+S \otimes \Lambda(n)_{1}+k z$, which is also denoted by $\hat{L}_{D}$. So we only need to consider the map $\bar{D}$. Let $\bar{D}$ have rank $r$. The (symmetric) 
bilinear form $(\cdot \mid \cdot)_{1}$ on $\Lambda(n)_{1}$ defined by

$$
(\lambda, \mu)_{1}:=\int \bar{D}(\lambda) \wedge \mu, \quad \lambda, \mu \in \Lambda(n)_{1}
$$

has radical of dimension $n-r$, since the pairing

$$
\int: \Lambda(n)_{n-1} \times \Lambda(n)_{1} \mapsto k
$$

is nondegenerate. Let $\lambda_{1}, \lambda_{2}, \ldots, \lambda_{n}$ be a basis of $\Lambda(n)_{1}$ such that $\lambda_{r+1}, \ldots, \lambda_{n}$ are contained in the radical of $(\cdot \mid \cdot)_{1}$, and $\lambda_{1}, \ldots, \lambda_{r}$ are chosen in such a way that the restriction of $(\cdot \mid \cdot)_{1}$ to the subspace $\sum_{i=1}^{r} k \lambda_{i}$ in the basis $\lambda_{1}, \ldots, \lambda_{r}$ has the following matrix representation:

$$
\begin{aligned}
& \left(\begin{array}{ccccc}
J & 0 & 0 & \cdots & 0 \\
0 & J & 0 & \cdots & 0 \\
0 & 0 & \ddots & & \vdots \\
\vdots & \vdots & & J & 0 \\
0 & 0 & \cdots & \cdots & 1
\end{array}\right) \text { for } r \text { odd } \\
& \left(\begin{array}{cccc}
J & 0 & \cdots & 0 \\
0 & J & \cdots & 0 \\
\vdots & \vdots & \ddots & \vdots \\
0 & 0 & \cdots & J
\end{array}\right) \text { for } r \text { even }
\end{aligned}
$$

where $J$ is the $2 \times 2$-matrix $J=\left(\begin{array}{ll}0 & 1 \\ 1 & 0\end{array}\right)$.

Remark. If $[p]$ stands for the smallest integer bigger than or equal to $p$, then the above means that $\left(\lambda_{2 i-1}, \lambda_{2 i}\right)_{1}=1$ for $i=1, \ldots,\left[\frac{r-1}{2}\right]$ (and in addition $\left(\lambda_{r}, \lambda_{r}\right)=1$ if $r$ is odd) with all other pairings being trivial.

Recall that $\hat{L}_{D}(\Lambda, c) \cong \hat{V}_{D}(\Lambda, c) / \hat{M}_{D}(\Lambda, c)$. Here $\hat{V}_{D}(\Lambda, c)=\operatorname{Ind}_{\hat{b}_{D}} \hat{L}_{\Lambda}$, where $\hat{\mathfrak{b}}_{D}=\mathfrak{b}+I_{D}+k z$ with $I_{D} \cdot v_{\Lambda}=0$, and $\hat{M}_{D}(\Lambda, c)$ is the unique maximal submodule in $\hat{V}_{D}(\Lambda, c)$. We have chosen $I_{D}$ to be a maximal isotropic subspace of $S \otimes \Lambda(n)_{1}$ containing $\mathrm{n}^{+} \otimes \Lambda(n)_{1}$ in the previous section. In addition to this we will now require $I_{D}$ to include the subspace generated by $\mathfrak{h} \otimes \lambda_{2}, \mathfrak{h} \otimes \lambda_{4}, \ldots, \mathfrak{h} \otimes \lambda_{2\left[\frac{r-1}{2}\right]}$, $\mathfrak{h} \otimes \lambda_{r+1}, \ldots, \mathfrak{h} \otimes \lambda_{n}$.

As before let $\left\{H_{l}, E_{l}, F_{l}\right\}, \alpha_{l} \in \Pi$, be some copy of $s l_{2}(k)$ inside the simple Lie algebra $S$.

Lemma 4.1. For $\lambda \in \Lambda(n)_{1}$ and $\left\{H_{l}, E_{l}, F_{l}\right\}$ a copy of $s_{2}(k)$ in $S$ we have the following identities in $\mathfrak{U}\left(\hat{L}_{D}\right)$, the universal enveloping superalgebra of $\hat{L}_{D}$ :

$$
\begin{aligned}
& \left(H_{l} \otimes \lambda\right) F_{l}^{j}=F_{l}^{j}\left(H_{l} \otimes \lambda\right)-2 j\left(F_{l} \otimes \lambda\right) F_{l}^{j-1}, \\
& \left(E_{l} \otimes \lambda\right) F_{l}^{n}=F_{l}^{n}\left(E_{l} \otimes \lambda\right)+n F_{l}^{n-1}\left(H_{l} \otimes \lambda\right)-n(n-1)\left(F_{l} \otimes \lambda\right) F_{l}^{n-2} .
\end{aligned}
$$

Proof. The first identity is an easy computation. The second identity follows from a similar computation using the first identity.

We have come to the main result of this section: 
Proposition 4.1. Suppose that $\Lambda\left(H_{l}\right)<r$, for some l. Then $\hat{L}_{D}(\Lambda, c)$ is infinite dimensional for $c \neq 0$.

Proof. Let $\Lambda\left(H_{l}\right)=n-1<r$. It is enough to show that $F_{l}^{m} v_{\Lambda} \notin \hat{M}_{D}(\Lambda, c)$ for all $m \in \mathbb{Z}_{+}$. Since $E_{l} F_{l}^{m} v_{\Lambda}=v(m) F_{l}^{m-1} v_{\Lambda}$ with $v(m) \neq 0$ for $m \neq n$, it is sufficient to show that $F_{l}^{n} v_{\Lambda} \notin \hat{M}_{D}(\Lambda, c)$. By assumption we have $n \leqq r$. It follows that

$$
k=\left[\frac{n-1}{2}\right] \leqq\left[\frac{r-1}{2}\right] .
$$

Therefore we can choose $\lambda_{i_{1}}, \lambda_{i_{2}}, \ldots, \lambda_{i_{k}}$ among the elements $\lambda_{2}, \lambda_{4}, \ldots, \lambda_{2\left[\frac{r-1}{2}\right]}$. Let $\left\{\mu_{i_{1}}, \mu_{i_{2}}, \ldots, \mu_{i_{k}}\right\}$ be the subset of $\left\{\lambda_{1}, \lambda_{2}, \ldots, \lambda_{n}\right\}$ such that $\left(\lambda_{i_{j}} \mid \mu_{i_{j}}\right)_{1}=1$ for all $j=1, \ldots, k$. By Lemma 4.1 and the fact that $\left\{H_{l} \otimes \lambda_{i_{1}}, H_{l} \otimes \lambda_{i_{2}}, \ldots, H_{l} \otimes \lambda_{i_{k}}\right\}$ $\subseteq I_{D}$, we obtain

$$
\begin{aligned}
\left(E_{l} \otimes \lambda_{i_{k}}\right) & \cdots\left(E_{l} \otimes \lambda_{i_{2}}\right)\left(E_{l} \otimes \lambda_{i_{1}}\right) F_{l}^{n} v_{\Lambda} \\
& =(-1)^{\frac{k(k+1)}{2}} n !\left(F_{l} \otimes \lambda_{i_{1}}\right)\left(F_{l} \otimes \lambda_{i_{2}}\right) \cdots\left(F_{l} \otimes \lambda_{i_{k}}\right) F_{l}^{n-2 k} v_{\Lambda} .
\end{aligned}
$$

Now if $n$ is even, then $2 k=n$, hence we are left with

$$
w_{1}=(-1)^{\frac{k(k+1)}{2}} n !\left(F_{l} \otimes \lambda_{i_{1}}\right)\left(F_{l} \otimes \lambda_{i_{2}}\right) \cdots\left(F_{l} \otimes \lambda_{i_{k}}\right) v_{\Lambda} \text {. }
$$

So

$$
\left(E_{l} \otimes \mu_{i_{k}}\right) \ldots\left(E_{l} \otimes \mu_{i_{2}}\right)\left(E_{l} \otimes \mu_{i_{1}}\right) w_{1}=(-1)^{\frac{k(k+1)}{2}} n !\left(\left(E_{l} \mid F_{l}\right)_{0}\right)^{k} c^{k} v_{\Lambda},
$$

and we are done.

If $n$ is odd, then $2 k+1=n$. Therefore

$$
\begin{aligned}
w_{2} & =\left(E_{l} \otimes \lambda_{i_{k}}\right) \cdots\left(E_{l} \otimes \lambda_{i_{2}}\right)\left(E_{l} \otimes \lambda_{i_{1}}\right) F_{l}^{n} v_{\Lambda} \\
& =(-1)^{\frac{k(k+1)}{2}} n !\left(F_{l} \otimes \lambda_{i_{1}}\right)\left(F_{l} \otimes \lambda_{i_{2}}\right) \cdots\left(F_{l} \otimes \lambda_{i_{k}}\right) F_{l} v_{\Lambda} .
\end{aligned}
$$

Now $2 k+1 \leqq r$ implies that $k<\left[\frac{r}{2}\right]$. So there exists some $\lambda_{t} \in\left\{\lambda_{1}, \lambda_{2}, \ldots, \lambda_{r}\right\}$ such that $\mathfrak{h} \otimes \lambda_{t} \nsubseteq I_{D}$. Therefore

$$
w_{3}=\left(E_{l} \otimes \lambda_{t}\right) w_{2}=(-1)^{\frac{k(k+3)}{2}} n !\left(F_{l} \otimes \lambda_{i_{1}}\right)\left(F_{l} \otimes \lambda_{i_{2}}\right) \cdots\left(F_{l} \otimes \lambda_{i_{k}}\right)\left(H_{l} \otimes \lambda_{t}\right) v_{\Lambda} .
$$

From this we get

$$
\left(E_{l} \otimes \mu_{i_{k}}\right) \cdots\left(E_{l} \otimes \mu_{i_{2}}\right)\left(E_{l} \otimes \mu_{i_{1}}\right) w_{3}=(-1)^{\frac{k(k+3)}{2}} n !\left(\left(E_{l} \mid F_{l}\right)_{0}\right)^{k} c^{k}\left(H_{l} \otimes \lambda_{t}\right) v_{\Lambda},
$$

which is a nonzero vector of weight $\Lambda$.

So the condition $\Lambda\left(H_{l}\right) \in \mathbb{Z}_{+}$for all $l$ is not sufficient for $\hat{L}_{D}(\Lambda, c)$ to be finite dimensional. From this proposition it follows that if $\hat{L}_{D}(\Lambda, c)$ is finite dimensional, then $\Lambda \geqq r \cdot \rho$, where $\rho=\frac{1}{2} \sum_{\alpha \in \Delta_{+}} \alpha$. The next section will show that this condition is also sufficient.

\section{Minimal Representations and the Character Formula}

Recall a few notations defined in the previous sections: $(\cdot 1 \cdot)_{0}$ denotes a symmetric invariant nondegenerate bilinear form of the simple Lie algebra $S . \bar{D}$ is the linear 
map obtained by restricting a fixed central derivation $D$ to $\Lambda(n)_{1}$, and it is assumed to have rank $r$. Furthermore $\bar{D}$ defines a bilinear form $(\cdot \mid \cdot)_{1}$ on $\Lambda(n)_{1}$, and $\lambda_{1}, \lambda_{1}, \ldots, \lambda_{n}$ form a "nice" basis of $\Lambda(n)_{1}$ with respect to this form, chosen in the previous section. Let $\hat{L}_{D}=S+S \otimes \Lambda(n)_{1}+k z$ and let $\hat{C}_{D}=S \otimes \Lambda(n)_{1}+k z$. $\hat{C}_{D}$ is a subalgebra of $\hat{L}_{D}$ and it is easily seen that $\hat{C}_{D}$ is a Clifford superalgebra. Let $I_{D}$ be the maximal isotropic subspace chosen in the previous section and let $|0\rangle$ be a nonzero vector on which $I_{D}$ acts trivially, and $z|0\rangle=c|0\rangle$ with $c \neq 0$. The unique nontrivial finite dimensional irreducible representation $\mathscr{F}$ of $\hat{C}_{D}$ is obtained by inducing $|0\rangle$ from $I_{D}+k z$ to $\hat{C}_{D}$, i.e.

$$
\mathfrak{F}=\operatorname{Ind}_{I_{D}+k z}^{\hat{C}_{D}}|0\rangle .
$$

We will now extend $\mathfrak{F}$ to a representation of $\hat{L}_{D}$. Choose an orthonormal basis $\left\{u_{1}, u_{2}, \ldots, u_{m}\right\}$ of $S$ with respect to $(\cdot \mid \cdot)_{0}$. Let $s \in S$. Suppose that $\left[s, u_{i}\right]=$ $\sum_{j=1}^{m} a_{i j} u_{j}$. We let $s$ act on $\mathfrak{F}$ as the following linear operator:

$$
\begin{aligned}
\phi(s)= & -\frac{1}{c} \sum_{i, j} a_{i j}\left(u_{i} \otimes \lambda_{2}\right)\left(u_{j} \otimes \lambda_{1}\right) \\
& -\frac{1}{c} \sum_{i, j} a_{i j}\left(u_{i} \otimes \lambda_{4}\right)\left(u_{j} \otimes \lambda_{3}\right)+\cdots \\
& -\frac{1}{c} \sum_{i, j} a_{i j}\left(u_{i} \otimes \lambda_{r-1}\right)\left(u_{j} \otimes \lambda_{r-2}\right) \\
& -\frac{1}{2 c} \sum_{i, j} a_{i j}\left(u_{i} \otimes \lambda_{r}\right)\left(u_{j} \otimes \lambda_{r}\right),
\end{aligned}
$$

for $r$ odd, and

$$
\begin{aligned}
\phi(s)= & -\frac{1}{c} \sum_{i, j} a_{i j}\left(u_{i} \otimes \lambda_{2}\right)\left(u_{j} \otimes \lambda_{1}\right) \\
& -\frac{1}{c} \sum_{i, j} a_{i j}\left(u_{i} \otimes \lambda_{4}\right)\left(u_{j} \otimes \lambda_{3}\right)+\cdots \\
& -\frac{1}{c} \sum_{i, j} a_{i j}\left(u_{i} \otimes \lambda_{r}\right)\left(u_{j} \otimes \lambda_{r-1}\right),
\end{aligned}
$$

if $r$ is even.

$\phi(s)$ is certainly well-defined. It remains to check that this definition makes $\mathfrak{F}$ into a representation of $\hat{L}_{D}$. We need to verify the following:

(i) $\left[s, u_{k}\right] \otimes \lambda_{j}=\left[\phi(s), u_{k} \otimes \lambda_{j t}\right] \forall k, j$.

(ii) If $t \in S$, then $\phi([s, t])=[\phi(s), \phi(t)]$.

Because of the way we have chosen our basis $\left\{\lambda_{1}, \lambda_{2}, \ldots, \lambda_{n}\right\}$, it suffices to check these identities in only 2 cases, namely $r=1$ and $r=2$. 
Case 1: $r=1$. Write $\tilde{u}_{i}$ for $u_{i} \otimes \lambda_{1}, i=1,2, \ldots, m$. In this case

$$
\phi(s)=-\frac{1}{c} \sum_{i, j} a_{i j} \tilde{u}_{i} \tilde{u}_{j} .
$$

We have two identities in $\operatorname{End}_{k}(\mathfrak{F})$ :

(a) $\left[\tilde{u}_{i} \tilde{u}_{j}, \tilde{u}_{k}\right]=c\left(\delta_{k j} \tilde{u}_{i}-\delta_{k i} \tilde{u}_{j}\right)$.

(b) $\left[\tilde{u}_{i} \tilde{u}_{j}, \tilde{u}_{k} \tilde{u}_{l}\right]=c\left(\delta_{i k} \tilde{u}_{l} \tilde{u}_{j}+\delta_{k j} \tilde{u}_{i} \tilde{u}_{l}-\delta_{l i} \tilde{u}_{k} \tilde{u}_{j}-\delta_{l j} \tilde{u}_{i} \tilde{u}_{k}\right)$.

We need to verify:

(i) $\phi\left(\left[s, \tilde{u}_{k}\right]\right)=\left[\phi(s), \tilde{u}_{k}\right]$.

(ii) If $t \in S$ and $\left[t, u_{i}\right]=\sum_{j} b_{i j} u_{j}$, then $\phi([s, t])=[\phi(s), \phi(t)]$.

(i) We compute both sides of the identity:

for the left-hand side we have

$$
\left.\phi\left(\left[s, \tilde{u}_{k}\right]\right)=\widetilde{\left[s, \tilde{u}_{k}\right.}\right]
$$

For the right-hand side we have

$$
\begin{aligned}
& {\left[\phi(s), \tilde{u}_{k}\right]=-\frac{1}{2 c} \sum_{i, j} a_{i j}\left[\tilde{u}_{i} \tilde{u}_{j}, \tilde{u}_{k}\right]} \\
& =-\frac{1}{2 c} \sum_{i, j} a_{i j} c\left(\delta_{k j} \tilde{u}_{i}-\delta_{k i} \tilde{u}_{j}\right) \quad \text { by (a) } \\
& =-\frac{1}{2} \sum_{i} a_{i k} \tilde{u}_{i}+\frac{1}{2} \sum_{j} a_{k j} \tilde{u}_{j} \\
& =\frac{1}{2} \sum_{i} a_{k i} \tilde{u}_{i}+\frac{1}{2} \sum_{j} a_{k j} \tilde{u}_{j} \\
& \left.=\sum_{j} a_{k j} \tilde{u}_{j}=\widetilde{\left[s, u_{k}\right.}\right] \text {. }
\end{aligned}
$$

(ii) As in (i) we compute both sides of the identity:

For the left-hand side we compute

$$
\begin{aligned}
{\left[[s, t], u_{i}\right] } & =-\left[t,\left[s, u_{i}\right]\right]+\left[s,\left[t, u_{i}\right]\right] \\
& =-\left[t, \sum_{j} a_{i j} u_{j}\right]+\left[s, \sum_{j} b_{i j} u_{j}\right] \\
& =\sum_{k}\left(\sum_{j} a_{j k} b_{i j}-a_{i j} b_{j k}\right) u_{k} .
\end{aligned}
$$

Thus

$$
\phi([s, t])=-\frac{1}{2 c} \sum_{i, k}\left(\sum_{j} a_{j k} b_{i j}-a_{i j} b_{j k}\right) \tilde{u}_{i} \tilde{u}_{k} .
$$


The right-hand side equals to

$$
\begin{aligned}
{[\phi(s), \phi(t)]=} & \frac{1}{4 c^{2}}\left[\sum_{i, j} a_{i j} \tilde{u}_{i} \tilde{u}_{j}, \sum_{k, l} b_{k l} \tilde{u}_{k} \tilde{u}_{l}\right] \\
= & \frac{1}{4 c^{2}} \sum_{i, j, k, l} a_{i j} b_{k l}\left[\tilde{u}_{i} \tilde{u}_{j}, \tilde{u}_{k} \tilde{u}_{l}\right] \\
= & \frac{1}{4 c^{2}} \sum_{i, j, k, l} a_{i j} b_{k l} c\left(\delta_{i k} \tilde{u}_{l} \tilde{u}_{j}+\delta_{k j} \tilde{u}_{i} \tilde{u}_{l}-\delta_{l i} \tilde{u}_{k} \tilde{u}_{j}-\delta_{l j} \tilde{u}_{i} \tilde{u}_{k}\right) \quad \text { by (b) } \\
= & \frac{1}{4 c} \sum_{i, j, l} a_{i j} b_{i l} \tilde{u}_{l} \tilde{u}_{j}+\frac{1}{4 c} \sum_{i, j, l} a_{i j} b_{j l} \tilde{u}_{i} \tilde{u}_{l} \\
& -\frac{1}{4 c} \sum_{i, j, k} a_{i j} b_{k i} \tilde{u}_{k} \tilde{u}_{j}-\frac{1}{4 c} \sum_{i, j, k} a_{i j} b_{k j} \tilde{u}_{i} \tilde{u}_{k} \\
= & -\frac{1}{4 c} \sum_{i, j, l} a_{i j} b_{l i} \tilde{u}_{l} \tilde{u}_{j}+\frac{1}{4 c} \sum_{i, j, l} a_{i j} b_{j l} \tilde{u}_{i} \tilde{u}_{l} \\
& -\frac{1}{4 c} \sum_{i, j, k} a_{i j} b_{k i} \tilde{u}_{k} \tilde{u}_{j}+\frac{1}{4 c} \sum_{i, j, k} a_{i j} b_{j k} \tilde{u}_{i} \tilde{u}_{k} \\
= & -\frac{1}{2 c} \sum_{i, j, k} a_{i j} b_{k i} \tilde{u}_{k} \tilde{u}_{j}+\frac{1}{2 c} \sum_{i, j, k} a_{i j} b_{j k} \tilde{u}_{i} \tilde{u}_{k} \\
= & -\frac{1}{2 c} \sum_{i, j, k} a_{j k} b_{i j} \tilde{u}_{i} \tilde{u}_{k}+\frac{1}{2 c} \sum_{i, j, k} a_{i j} b_{j k} \tilde{u}_{i} \tilde{u}_{k} \\
= & -\frac{1}{2 c} \sum_{i, j, k}\left(\sum_{j} a_{j k} b_{i j}-a_{i j} b_{j k}\right) \tilde{u}_{i} \tilde{u}_{k} .
\end{aligned}
$$

This proves (ii) for $r=1$. Therefore $\mathfrak{F}$ is a representation of $\hat{L}_{D}$ in this case.

Case 2: $r=2$. Write $\tilde{u}_{i}$ for $u_{i} \otimes \lambda_{1}$ and $\tilde{u}_{i}^{*}$ for $u_{i} \otimes \lambda_{2}$. In this case we have

$$
\phi(s)=-\frac{1}{c} \sum_{i, j} a_{i j} \tilde{u}_{i}^{*} \tilde{u}_{j}
$$

To verify (i) and (ii) we use

$\left(\mathrm{a}^{\prime}\right)\left[\tilde{u}_{i}^{*} \tilde{u}_{j}, \tilde{u}_{k}\right]=-\delta_{i k} c \tilde{u}_{j}$.

(b') $\left[\tilde{u}_{i}^{*} \tilde{u}_{j}, \tilde{u}_{k}^{*} \tilde{u}_{l}\right]=\delta_{k j} c \tilde{u}_{i}^{*} \tilde{u}_{l}-\delta_{l i} c \tilde{u}_{k}^{*} \tilde{u}_{j}$.

The verification of (i) and (ii) in this case are very similar to the one in the case when $r=1$. One uses the $\left(a^{\prime}\right)$ to show (i), and $\left(b^{\prime}\right)$ to show (ii). We will omit the details.

It follows that $\mathfrak{F}$ is an irreducible representation of $L_{D}$. We know from previous sections that $\mathfrak{F}$ is a highest weight module; thus we can calculate its highest weight. For this we need to specify an orthonormal basis for $S$. We choose an arbitrary orthonormal basis for $\mathfrak{h}$. Let $\Delta_{+}$be our set of positive roots. For each $\alpha \in \Delta_{+}$we let $e_{\alpha}$ be a nonzero element in the $\alpha$-weight space of $S$. Set $f_{\alpha}$ to be an element in the opposite weight space such that $\left(e_{\alpha} \mid f_{\alpha}\right)_{0}=1$. Define $u_{\alpha}:=\frac{1}{\sqrt{2}}\left(e_{\alpha}+f_{\alpha}\right)$ and $u_{\alpha}^{\prime}:=\frac{i}{\sqrt{2}}\left(e_{\alpha}-f_{\alpha}\right)$, where $i^{2}=-1$. Let $H_{l}, E_{l}, F_{l}, \alpha_{l} \in \Pi$, be some copy of $s l_{2}(k)$ in $S$, 
as in the previous sections. We want to compute the action of $H_{l}$ on the highest weight vector $|0\rangle$ in $\mathfrak{F}$.

One easily computes $\left[H_{l}, u_{\alpha}\right]=-i \alpha\left(H_{l}\right) u_{\alpha}^{\prime}$ and $\left[H_{l}, u_{\alpha}^{\prime}\right]=i \alpha\left(H_{l}\right) u_{\alpha}$. From this it follows that

$$
\begin{aligned}
\phi\left(H_{l}\right)|0\rangle= & \left\{-\frac{1}{c} \sum_{\alpha \in \Delta_{+}}-i \alpha\left(H_{l}\right)\left(u_{\alpha} \otimes \lambda_{2}\right)\left(u_{\alpha}^{\prime} \otimes \lambda_{1}\right)\right. \\
& +i \alpha\left(H_{l}\right)\left(u_{\alpha}^{\prime} \otimes \lambda_{2}\right)\left(u_{\alpha} \otimes \lambda_{1}\right)-\cdots \\
& -\frac{1}{c} \sum_{\alpha \in \Delta_{+}}-i \alpha\left(H_{l}\right)\left(u_{\alpha} \otimes \lambda_{r-1}\right)\left(u_{\alpha}^{\prime} \otimes \lambda_{r-2}\right) \\
& +i \alpha\left(H_{l}\right)\left(u_{\alpha}^{\prime} \otimes \lambda_{r-1}\right)\left(u_{\alpha} \otimes \lambda_{r-2}\right) \\
& -\frac{1}{2 c} \sum_{\alpha \in \Delta_{+}}-i \alpha\left(H_{l}\right)\left(u_{\alpha} \otimes \lambda_{r}\right)\left(u_{\alpha}^{\prime} \otimes \lambda_{r}\right) \\
& \left.+i \alpha\left(H_{l}\right)\left(u_{\alpha}^{\prime} \otimes \lambda_{r}\right)\left(u_{\alpha} \otimes \lambda_{r}\right)\right\}|0\rangle,
\end{aligned}
$$

for $r$ odd, and

$$
\begin{aligned}
\phi\left(H_{l}\right)|0\rangle= & \left\{-\frac{1}{c} \sum_{\alpha \in \Delta_{+}}-i \alpha\left(H_{l}\right)\left(u_{\alpha} \otimes \lambda_{2}\right)\left(u_{\alpha}^{\prime} \otimes \lambda_{1}\right)\right. \\
& +i \alpha\left(H_{l}\right)\left(u_{\alpha}^{\prime} \otimes \lambda_{2}\right)\left(u_{\alpha} \otimes \lambda_{1}\right)-\cdots \\
& -\frac{1}{c} \sum_{\alpha \in \Delta_{+}}-i \alpha\left(H_{l}\right)\left(u_{\alpha} \otimes \lambda_{r}\right)\left(u_{\alpha}^{\prime} \otimes \lambda_{r-1}\right) \\
& \left.+i \alpha\left(H_{l}\right)\left(u_{\alpha}^{\prime} \otimes \lambda_{r}\right)\left(u_{\alpha} \otimes \lambda_{r-1}\right)\right\}|0\rangle
\end{aligned}
$$

for $r$ even.

Using the facts that $\left(e_{\alpha} \otimes \lambda_{j}\right)|0\rangle=0$ and $\left(f_{\alpha} \otimes \lambda_{j}\right)^{2}=0, \forall j$, we find that

$$
\phi\left(H_{l}\right)|0\rangle=r \cdot \frac{1}{2} \sum_{\alpha \in \Delta_{+}} \alpha\left(H_{l}\right)=r .
$$

Hence $\mathfrak{F}=\hat{L}_{D}(r \rho, c)$, where $\rho=\frac{1}{2} \sum_{\alpha \in \Delta_{+}} \alpha$.

Now let $\Lambda \geqq r \rho$. Let $L(\Lambda-r \rho)$ be the finite dimensional irreducible representation of $S$ with highest weight $\Lambda-r \rho$. We can extend $L(\Lambda-r \rho)$ to a representation of $\hat{L}_{D}$ by letting $\hat{C}_{D}$ act trivially. We can form the tensor product of $\hat{L}_{D}(r \rho, c)$ and $L(\Lambda-r \rho)$.

Proposition 5.1. $\hat{L}_{D}(\Lambda, c) \cong \hat{L}_{D}(r \rho) \otimes L(\Lambda-r \rho)$.

Proof. Since $\hat{L}_{D}(r \rho, c) \otimes L(\Lambda-r \rho)$ has highest weight $\Lambda$ and $z$ acts as the scalar $c$, it suffices to show that this module is irreducible. This is true, since $\hat{L}_{D}(r \rho, c)$ is an irreducible $\hat{C}_{D}$-module and $L(\Lambda-r \rho)$ is an irreducible $S$-module. Choose a homogeneous basis $v_{1}, v_{2}, \ldots, v_{m}$ for $\hat{L}_{D}(r \rho, c)$ and a basis $w_{1}, w_{2}, \ldots, w_{t}$ for $L(\Lambda-r \rho)$. An arbitrary homogeneous nonzero element of $\hat{L}_{D}(r \rho, c) \otimes L(\Lambda-r \rho)$ has the form

$$
x=\sum_{i, j} a_{i j}\left(v_{i} \otimes w_{j}\right), \quad a_{i j} \in k .
$$


Note that since $x$ is homogeneous, only those $v_{i}$ 's of the same parity can have nonzero $a_{i j}$ 's. We can assume that $a_{11} \neq 0$. By Burnside's theorem for superalgebras (see below) we may choose some $c \in \mathfrak{U}\left(\mathscr{C}_{D}\right)$ such that $c v_{1}=v_{1}$ and $c v_{j}=0$ for $j \neq 1$ and $v_{j}$ of the same parity as $v_{1}$. Thus $(c \otimes 1) x=a_{11}\left(v_{1} \otimes w\right)$, for some $0 \neq w \in L(\Lambda-r \rho)$. Now $v_{1} \otimes w$ generates the whole module, since for each $i, j$ we can find some $s_{j} \in \mathfrak{U}(S)$ and $c_{i} \in \mathfrak{U}\left(\hat{C}_{D}\right)$ such that $s_{j} w=w_{j}$ and $c_{i} v_{1}=v_{i}$. Then $\left(1 \otimes s_{j}\right)\left(c_{i} \otimes 1\right) v_{1} \otimes w=v_{i} \otimes w_{j}+s_{j} v_{i} \otimes w$. But there exists some $c^{\prime} \in \mathfrak{U}\left(\hat{C}_{D}\right)$ such that $c^{\prime} v_{1}=s_{j} v_{i}$. Hence $\left\{\left(1 \otimes s_{j}\right)\left(c_{i} \otimes 1\right)-\left(c^{\prime} \otimes 1\right)\right\} v_{1} \otimes w=v_{i} \otimes w_{j}$.

Remark. Let $p$ denote the dimension of the Cartan subalgebra $\mathfrak{h}$. Since the highest weight of $\mathfrak{F}$ is $r \rho$, the character of $\hat{L}_{D}(r \rho, c)$ is

$$
\operatorname{ch} \hat{L}_{D}(r \rho, c)=2^{\left[\frac{r p}{2}\right]} e^{r \rho} \prod_{\alpha \in \Delta_{+}}\left(1+e^{-\alpha}\right)^{r} .
$$

Remark. Burnside's theorem for superalgebras is similar to the one for algebras. It says the following: let $B$ be a $k$-superalgebra and let $\left(\pi_{B}, V_{B}\right)$ be a simple $B$-module. Then $\pi_{B}(B) \cong \operatorname{End}_{D}\left(V_{B}\right)$, where $D=\operatorname{End}_{B}\left(V_{B}\right)$ is either $k$ or $C(1)$, the Clifford superalgebra in one indeterminate.

Summarizing our results we obtain the following:

Theorem 5.1. Let $D$ be a central derivation of degree $n-2$ such that the restriction of $D$ to $\Lambda(n)_{1}$ has rank $r$. Let $\hat{L}_{D}$ be the corresponding central extension of $S \otimes \Lambda(n)$ and $\hat{L}_{D}(\Lambda, c)$ be the unique irreducible highest weight representation of $\hat{L}_{D}$ with highest weight $\Lambda$ such that $z$ acts as a nonzero scalar $c \in k$. Then $\hat{L}_{D}(\Lambda, c)$ is finite dimensional if and only if $\Lambda \geqq r \rho$ and its character is given by

$$
\operatorname{ch} \hat{L}_{D}(\Lambda, c)=2^{\left[\frac{r p}{2}\right]} e^{(r-1) \rho} \sum_{w \in W} \varepsilon(w) e^{w(\Lambda-(r-1) \rho)} \prod_{\alpha \in A_{+}} \frac{\left(1+e^{-\alpha}\right)^{r}}{\left(1-e^{-\alpha}\right)},
$$

where $W$ is the Weyl group, and $p$ is the dimension of the Cartan subalgebra of $S$.

Proof. The character formula follows from Proposition 5.1, the remark following it and the Weyl character formula.

Acknowledgements. I would like to thank my Ph.D. advisor Victor G. Kač of Massachusetts Institute of Technology for the many stimulating discussions and for the valuable guidance he has provided me with so far. I also express my gratitude to the Harvard Mathematics Department for supporting me while this paper was written.

\section{References}

[B] Block, R.E.: Determination of differentiably simple rings with a minimal ideal. Ann. Math. 90, 2, 433-459 (1969)

[C] Cheng, S.-J.: Differentiably simple Lie superalgebras. Dissertation, Harvard Univ. (1993)

[K1] Kač, V.G.: Lie superalgebras. Adv. in Math. 26 (1977)

[K2] Kač, V.G.: Infinite dimensional Lie algebras. (3rd ed.) Cambridge: Cambridge University Press 1990

[K3] Kač, V.G., Todorov, I.T.: Superconformal current algebras and their representations. Comm. Math. Phys. 102, 337-347 (1985) 